\title{
Indicadores financeiros e não financeiros e a qualidade da educação superior das universidades federais brasileiras
}

\author{
Financial and non-financial indicators and the quality of higher education in the \\ brazilian federal universities
}

\author{
Kleber Morais de Sousa ${ }^{1}$ \\ Antônio André Cunha Callado²
}

\begin{abstract}
Resumo
Esta pesquisa teve por objetivo investigar a relação dos indicadores financeiros e não financeiros com a qualidade do ensino superior das universidades federais brasileiras. A pesquisa foi realizada por meio de levantamento documental dos indicadores de desempenho definidos pelo Tribunal de Contas da União na Decisão n. ${ }^{\circ} 408 / 2002$, mensurados por 50 universidades brasileiras no ano de 2015. Os indicadores de qualidade foram obtidos nas três avaliações realizadas pelo Instituto Nacional de Estudos e Pesquisas Educacionais Anísio Teixeira (INEP): Índice Geral de Cursos (IGC), Conceito Preliminar de Curso (CPC) e Exame Nacional de Desempenho de Estudantes (Enade). O tratamento dos dados foi realizado por meio de análise de correlação e teste de Spearman para obtenção das conclusões do estudo. Os principais resultados evidenciam a qualificação dos professores, o fortalecimento dos programas de pós-graduação e o volume de recursos gastos por aluno estão, positivamente e estatisticamente, correlacionados com melhores indicadores de qualidade das universidades federais brasileiras.
\end{abstract}

Palavras-chave: Indicadores; Qualidade; Educação Superior.

\begin{abstract}
This article aims to investigate the relation between financial and non-financial indicators and the quality of higher education of the Brazilian federal universities. The research was performed through documental survey with performance indicators defined by Nacional Count Court at decision n. 408/2002 with 50 Brazilian universities at 2015. The quality indicators were collected from tree evaluations of the National Institute of Study and Educational Research Anísio Teixeira (INEP); General Indicator of Courses (GIC); Preliminary Conceptual of Courses (PCC); and National Exam of Student Performances (NESP). The treatment of data was performed by correlation analysis and Spearman Test to obtain the conclusions of study. The mains results showed that the qualification of Professors, the strengthening of the graduate program and the amount of expenditures by student are positive and statistical correlation with the higher quality indicators of Brazilian Federal Universities.
\end{abstract}

Keywords: Indicators; Quality; Higher Education.

\section{Introdução}

O enfrentamento dos problemas do sistema educacional de ensino superior brasileiro no decorrer das últimas três décadas passou por diferentes caminhos. A expansão da oferta de matrículas, que saltou de 3.036.113, em 2001, para 6.379.299, em 2010, reflete a prioridade que a deficiência de acesso teve no período (BARROS, 2015). Entretanto esse crescimento careceu de qualidade no ensino. Atualmente, a

Doutorando em Ciências Contábeis pela Universidade Federal da Paraíba (UFPB)

Professor Assistente da Universidade Federal Rural de Pernambuco (UFRPE). Brasil. Afiliação: Universidade Federal Rural de Pernambuco (UFRPE)

Universidade Federal da Paraíba (UFPB). Lattes: http://lattes.cnpq.br/0980086519281168. Email: kleberfinancas@gmail.com

2 Doutor em Administração na área de Estratégias Empresariais (linha de pesquisa em Finanças) pelo Programa de Pós-Graduação em Administração da Universidade Federal da Paraíba (2001) e Doutor em Administração na área de Gestão Organizacional (linha de pesquisa em Finanças) pelo Programa de Pós-Graduação em Administração da Universidade Federal de Pernambuco (2009). Professor Titular da Universidade Federal Rural de Pernambuco (UFRPE). Brasil. Afiliação: Universidade Federal Rural de Pernambuco (UFRPE). Email: andrecallado@yahoo.com.br 
qualidade do ensino é avaliada pelo Sistema Nacional de Avaliação da Educação Superior (SINAES) que, em suma, avalia o desempenho dos alunos, cursos e instituições de ensino superior no Brasil (COLOSSI; CONSENTINO; QUEIROZ, 2017).

$\mathrm{Na}$ década de 1990, a gestão pública passou por esforços no sentido de adotar uma estrutura gerencial focada, principalmente, em metas e objetivos, ao invés do exclusivo atendimento aos processos burocráticos. Essa iniciativa ficou conhecida como Reforma Gerencial do Estado e teve como balizador o Plano Diretor da Reforma do Aparelho do Estado, criado em 1995, durante o governo de Fernando Henrique Cardoso (FHC). Contudo, a reforma não foi plenamente concretizada. As instituições adotaram em diferentes níveis as diretrizes estabelecidas no plano, de modo que o grau de utilização dos instrumentos gerenciais é discrepante, tanto dentre as instituições públicas quanto dentre os níveis federativos (BRESSER-PEREIRA, 2000; 2009).

No âmbito organizacional, as universidades necessitam de aprimoramento dos seus instrumentos de gestão para alcançar o nível de qualidade desejado das políticas públicas de educação. Nesse contexto, instrumentos de mensuração de desempenho são fundamentais para direcionar as ações necessárias para a concretização das metas e dos objetivos almejados. Os gestores das universidades federais brasileiras já são obrigados a apresentar relatórios de gestão que evidenciem os resultados obtidos em cada exercício financeiro, ou seja, as normas já existem, mas é necessário o aprimoramento dos indicadores e a investigação das relações existentes entre eles.

Estudos desenvolvidos na investigação da utilização dos indicadores de desempenho, financeiros ou não financeiros, são principalmente focados nas organizações do setor privado, como: Callado, Callado e Holanda (2008), Callado, Callado e Andrade (2009), Marquezan, Diehl e Alberton (2013), Curcino, Lemes e Botinha (2014), Vignochi, Gonçalo e Lezana (2014) e Vargas et al (2016). Em menor quantidade, há estudos que investigam a utilização de indicadores de desempenho no setor público, por exemplo: Marengo e Diehl (2011), Helou Filho e Otani (2007) e Silva, Bartoluzzio e Anjos (2017). A utilização de indicadores de desempenho no setor público possui diferenças em relação ao setor privado, porque a finalidade das instituições públicas é a satisfação da sociedade com serviços públicos, ao contrário das empresas, que têm o lucro como foco (BEHN, 2003).

Assim, esta pesquisa busca contribuir com o preenchimento parcial dessa lacuna, especificamente em relação às instituições públicas de ensino superior do âmbito federal. Por conseguinte, o estudo teve por objetivo investigar a relação dos indicadores financeiros e não financeiros com a qualidade do ensino superior nas universidades federais brasileiras.

O estudo utilizou uma metodologia descritiva de abordagem quantitativa. Os dados dos indicadores financeiros e não financeiros foram obtidos nos relatórios de gestão publicados pelas universidades federais brasileiras relativos ao exercício de 2015. A mensuração da qualidade foi realizada por meio dos componentes individualizados dos indicadores de qualidade do Índice Geral de Cursos (IGC), do Conceito Preliminar de Curso (CPC) e do Exame Nacional de Desempenho de Estudantes (ENADE), obtidos na base de dados do Instituto Nacional de Estudos e Pesquisas Educacionais Anísio Teixeira (INEP), correspondente ao exercício de 2015.

Além desta introdução, o estudo estrutura-se em mais quatro seções. A segunda seção trata do referencial teórico direcionado a apresentação dos fundamentos teóricos da utilização de indicadores de desempenho e qualidade do ensino superior. A terceira seção descreve a metodologia utilizada para concretização do estudo. A quarta parte apresenta os resultados obtidos na pesquisa e, por último, a quinta seção apresenta a conclusão do estudo.

\section{Referencial Teórico}

\subsection{Indicadores de desempenho}

Indicadores de desempenho são instrumentos utilizados para quantificar a eficiência ou eficácia de uma tomada de decisão, de modo a avaliar como estão sendo realizadas as atividades, inclusive em comparação 
com a metas preestabelecidas (NEELY et al, 1996, MACARTHUR, 1996). A mensuração do desempenho por meio de indicadores envolve a produção de informações capazes de direcionar ações preventivas e corretivas, por meio da aquisição, coleta, classificação, análise, interpretação e disseminação dos dados apropriados (NEELY et al, 1998).

Nesse sentido, os indicadores de desempenho fornecem informações capazes de subsidiar a tomada de decisão nos processos de planejamento e controle, portanto, sendo necessário, portanto, que os indicadores façam parte dos instrumentos e mecanismos cotidianos de planejamento e controle das organizações (SINK; TUTTLE, 1993; NEELY et al, 1997). Não existe, entretanto, um padrão de uso dos indicadores nas organizações. Os indicadores devem ser flexíveis, ou seja, devem ser avaliados de acordo com o contexto das atividades que são desempenhadas, alinhados aos objetivos estratégicos, e devem considerar as restrições da empresa (NEELY, 1999). Assim, a definição dos indicadores não é uma tarefa simples, porque depende da complexidade do processo, da importância da atividade em relação às metas estabelecidas e da expectativa de uso das informações no processo gerencial (CALLADO; CALLADO; ALMEIDA, 2008), pois os indicadores de desempenho devem ser adaptados ao contexto organizacional (NEELY et al, 1997).

Em termos de resultado, a utilização de indicadores de desempenho é o método mais efetivo e menos oneroso de avaliar e modificar o comportamento das pessoas quando definidos de forma adequada (HRONEC; ANDERSEN, 1994). A sistematização de indicadores de desempenho pode, principalmente, favorecer a avaliação de desempenho em longo prazo, o aumento da competitividade e a atração de investimentos (KOTANE; KUZMINA-MERLINO, 2011). Além disso, a avaliação do desempenho exerce grande influência nas decisões estratégicas e operacionais, no alcance dos fins almejados e na criação de valor para os acionistas (FOLAN; BROWNE, 2005; MARTINS et al, 2013).

Por outro lado, a utilização de indicadores de desempenho possui limitações. A dificuldade de se desenvolver indicadores de desempenho que representem convincentemente as projeções em longo prazo (AAKER, 2001), a complexidade da utilização de um indicador para representar a performance, pois um indicador isolado não é capaz de abranger as distintas áreas da organização (KAPLAN; NORTON, 1992), e a restrição dos indicadores financeiros em apenas evidenciar a perspectiva histórica (NEELY, 2002) são alguns dos aspectos que necessitam cautela no uso de indicadores e na elaboração de um sistema de mensuração de desempenho.

Nesse sentido, os indicadores de desempenho passaram a evidenciar não somente os aspectos financeiros, mas também os não financeiros, porque já existe unanimidade no campo de pesquisa de que as avaliações não financeiras devem ser utilizadas como instrumento de equilíbrio da ênfase financeira (NEELY, 2002). Os indicadores não financeiros permitem melhor ajustamento e conexão entre o passado, o presente e o planejamento para o futuro (VARGAS et al, 2016). Além disso, os indicadores não financeiros permitem redução dos conflitos entre gestores e empregados, por permitirem melhor avaliação do esforço dedicado pelos empregados, favorecem o fornecimento de informações relacionadas a comportamento, influência e mudanças nas necessidades de competência (POINCELOT; WEGMANN, 2008).

\subsection{Avaliação de desempenho no setor público}

No setor público, a ampliação das funções do Estado resultou na necessidade de a gestão pública deixar de ser um compilador de dados e passar a ser um gestor com capacidade de definição e alinhamento estratégico para obtenção dos resultados necessários nas políticas públicas. Em outras palavras, a gestão, que antes era conduzida apenas com preocupação política, passa a dar espaço para a gestão profissional, em que a definição de estratégias, objetivos, metas e análise de indicadores são fundamentais para o melhor desenvolvimento da oferta de serviços públicos à população (LUNKES; GASPARETTO; SCHNORRENBERG, 2010).

Além disso, a sociedade tem exigido cada vez mais eficiência na utilização dos recursos dos impostos arrecadados e a maior participação social tem fortalecido os meios de controle, de modo que a utilização de indicadores de desempenho tem se tornado instrumentos importantes no processo de gestão e prestação de contas (CATELLI; SANTOS, 2004). Não basta os gestores públicos apresentarem os valores arrecadados e gastos pelos demonstrativos fiscais, pois torna-se necessário avaliar a quantidade e a qualidade dos 
serviços prestados à sociedade. Nesse sentido, é fundamental detalhar os caminhos escolhidos e apresentar mensurações que evidenciem claramente os resultados alcançados. Assim, além do aperfeiçoamento da gestão, os indicadores de desempenho no setor público podem ser instrumentos que auxiliam no processo de accountability à medida que que são fornecidos aos cidadãos.

Esforços no sentido de aperfeiçoamento da gestão foram realizados no decorrer das últimas décadas. A iniciativa de utilizar instrumentos de gestão do setor privado no setor público veio como resposta ao esgotamento do modelo burocrático da administração pública, que privilegiava os procedimentos administrativos e o cumprimento da lei. A reforma da gestão pública, ocorrida na década de 1990, teve o propósito de aumentar a responsabilidade dos gestores, de mudar o enfoque de controle de processos para resultados e de estabelecer maior rigidez no detalhamento do desempenho. Esse processo visou, principalmente, a melhoria da eficiência e efetividade do setor público e ficou conhecido como a Nova Administração Pública (BRESSER-PEREIRA, 2000; 2009).

É importante atentar que as técnicas do setor privado não podem ser utilizadas da mesma maneira no setor público. A perspectiva do setor privado é a maximização do lucro, ao passo que o setor público foca no cumprimento da função social, principalmente com a oferta de bens públicos, ou seja, as ferramentas do setor privado precisam passar por adaptações para que exerçam adequadamente suas funções (MARINI, 1999). Segundo Behn (2003), a avaliação de desempenho no setor público busca, especialmente, atender aos objetivos descritos no Quadro 1, a seguir:

Quadro 1 - Objetivos específicos e distintos para os gestores medirem desempenho

\begin{tabular}{|c|c|}
\hline \multicolumn{2}{|c|}{ Avaliação de Desempenho } \\
\hline Questão Objetivo & Desempenho medido pode ajudar a responder? \\
\hline Avaliar & Como os gestores sabem se o órgão público está desempenhando bem as suas funções? \\
\hline Controlar & Como os gestores podem assegurar que seus colaboradores estão fazendo certo? \\
\hline Orçar & $\begin{array}{l}\text { Como os gestores determinam em que projetos, programas e pessoas da organização } \\
\text { devem aplicar os recursos públicos? }\end{array}$ \\
\hline Motivar & $\begin{array}{l}\text { Como os gestores podem motivar os servidores e todas as partes interessadas a } \\
\text { melhorar o desempenho? }\end{array}$ \\
\hline Promover & $\begin{array}{l}\text { Como os gestores podem convencer a esfera política superior, os legisladores, as } \\
\text { partes interessadas, a mídia e a sociedade de que a organização está fazendo um bom } \\
\text { trabalho? }\end{array}$ \\
\hline Comemorar & Que realizações da organização são merecedoras de eventos de comemoração? \\
\hline Aprender & Como saber por que algumas coisas funcionam e outras não? \\
\hline Melhorar & $\begin{array}{l}\text { O que tem que ser feito para converter a medição de desempenho em melhorias } \\
\text { organizacionais? }\end{array}$ \\
\hline
\end{tabular}

Fonte: Behn (2003). 
Assim, o Quadro 1 evidencia que utilização de indicadores no setor público pode ser um forte instrumento para o aperfeiçoamento da gestão pública, o melhoramento da transparência e o aumento da compreensão do desempenho das ações públicas pela sociedade. Em um cenário de organizações públicas brasileiras que carecem de melhor consolidação e institucionalização dos métodos de governança pública (MATIASPEREIRA, 2010), a utilização de indicadores de desempenho é elemento que pode auxiliar nesse processo.

No setor público, os indicadores sociais são também considerados indicadores não financeiros, ou denominados de indicadores de nível de vida (COSTA; CASTANHAR, 2003). Os indicadores não financeiros exercem papel fundamental nas etapas de elaboração e implementação das políticas públicas nas diversas áreas, educação, moradia, saúde, emprego, entre tantas outras (HELOU FILHO; OTANI, 2007). Na área de educação, as universidades federais brasileiras possuem instrumentos já institucionalizados de gestão e controle das ações desenvolvidas. Portanto, é possível avaliar as relações existentes entre os indicadores de desempenho.

Diante da diversidade de formas de mensuração, o Tribunal de Contas da União (TCU) padronizou a mensuração dos indicadores de desempenho por meio da Decisão n. ${ }^{\circ} 408 / 2002$. A decisão estabeleceu 12 (doze) indicadores financeiros e não financeiros que devem ser mensurados anualmente e compor o relatório de gestão das universidades, que são: 1) Custo corrente com HU/Aluno Equivalente; 2) Custo corrente sem HU/Aluno Equivalente; 3) Aluno tempo integral/Professor equivalente; 4) Aluno tempo integral/ Funcionário equivalente com $\mathrm{HU}$; 5) Aluno tempo integral/Funcionário equivalente sem $\mathrm{HU}$; 6) Funcionário equivalente com HU/Professor equivalente; 7) Funcionário equivalente sem HU/Professor equivalente; 8) Grau de participação estudantil (GPE); 9) Grupos de envolvimento discente com Pós-Graduação (CEPG); 10) Conceito Capes/MEC para Pós-Graduação; 11) Índice de qualificação do corpo docente (IQCD); e 12) Taxa de sucesso na graduação (TSG).

Por outro lado, a avaliação da qualidade do ensino superior, no âmbito dos alunos, dos cursos e das universidades de ensino superior, é realizada pelo Sistema Nacional de Avaliação da Educação Superior (SINAES). Os indicadores utilizados são, principalmente, o Índice Geral de Cursos (IGC); o Conceito Preliminar de Curso (CPC); o Indicador de Diferença entre os Desempenhos Observado e Esperado (IDD); e o Exame Nacional de Desempenho de Estudantes (Enade) (BRASIL, 2015).

\section{Metodologia}

A metodologia utilizada é descritiva e de abordagem quantitativa. A coleta dos dados necessários para realização da pesquisa foi realizada em duas fontes. Os dados dos indicadores financeiros e não financeiros foram obtidos por meio dos relatórios de gestão das 50 universidades federais brasileiras, relativos ao exercício de 2015. Os relatórios de gestão foram coletados no site de cada uma das universidades. Em seguida, foi realizada a leitura dos relatórios com o propósito de coletar os indicadores financeiros e não financeiros, conforme definido pelo TCU. Os dados obtidos foram tabulados em planilhas eletrônicas.

A qualidade do ensino das universidades foi mensurada por meio dos componentes individualizados dos indicadores de qualidade do Índice Geral de Cursos (IGC), do Conceito Preliminar de Curso (CPC) e do Exame Nacional de Desempenho de Estudantes (Enade). Esses dados foram coletados na base de dados do Instituto Nacional de Estudos e Pesquisas Educacionais Anísio Teixeira (INEP), também correspondente ao exercício financeiro de 2015. O estudo não utilizou os indicadores gerais (IGC, CPC, IDD) por considerar que as ponderações e métodos de agregação acabam por reduzir o caráter informacional dos componentes avaliados. Assim, foram utilizados os componentes que formam os indicadores de qualidade mensurados pelo Sinaes. Os 12 indicadores primários utilizados, de acordo com a Decisão TCU n. ${ }^{\circ}$ 408/2002, estão descritos no Quadro 2: 
Quadro 2 - Indicadores primários de desempenho utilizados nas universidades federais -Decisão TCU n. ${ }^{\circ}$ $408 / 2002$.

\begin{tabular}{|c|c|c|}
\hline$n$ & Indicador & Fórmula \\
\hline \multirow{2}{*}{1} & \multirow{2}{*}{ Custo corrente com HU/Aluno equivalente } & Custo Corrente \\
\hline & & $\mathrm{A}_{G} \mathrm{E}+\mathrm{A}_{\mathrm{PG}} \mathrm{TI}+\mathrm{A}_{\mathrm{R}} \mathrm{TI}$ \\
\hline \multirow{2}{*}{2} & \multirow{2}{*}{ Custo corrente sem HU/Aluno equivalente } & Custo Corrente $\left.{ }^{*}\right)$ \\
\hline & & $\overline{A_{G} E+A_{P G} T I+A_{R} T I}$ \\
\hline 3 & Aluno tempo integral/Professor equivalente & 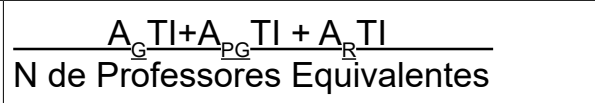 \\
\hline 4 & Aluno tempo integral/Funcionário equivalente com $\mathrm{HU}$ & $\frac{\mathrm{A}_{\mathrm{G}}}{\mathrm{N} \text { de Funcionários Equivalentes }}$ \\
\hline 5 & Aluno tempo integral/Funcionário equivalente sem $\mathrm{HU}$ & $\frac{\mathrm{A}_{G}}{\mathrm{~N} \text { de Funcionários Equivalentes }}$ \\
\hline 6 & Funcionário equivalente com HU/Professor equivalente & $\frac{\text { No de Funcionários Equivalentes }}{\text { No de Professores Equivalentes }}$ \\
\hline 7 & Funcionário equivalente sem HU/Professor equivalente & $\frac{\text { No de Funcionários Equivalentes }\left(^{*}\right)}{\text { No de Professores Equivalentes }}$ \\
\hline 8 & Grau de participação estudantil (GPE) & $\frac{A_{G}}{A_{G}} \underline{T I}$ \\
\hline 9 & Grupos de envolvimento discente com pós-graduação (CEPG) & $\frac{A_{P G}}{A_{G}+A_{P G}}$ \\
\hline \multirow{2}{*}{10} & \multirow{2}{*}{ Conceito Capes/MEC para pós-graduação } & $\begin{array}{c}\sum \text { Conceito de todos os programas de } \\
\text { pós-graduação }\end{array}$ \\
\hline & & No de programas de pós-graduação \\
\hline 11 & Índice de qualificação do corpo docente (IQCD) & $\frac{(5 D+3 M+2 E+G)}{(D+M+E+G)}$ \\
\hline 12 & Taxa de sucesso na graduação (TSG) & $\begin{array}{l}\text { No de Diplomados }\left(\mathrm{N}_{\mathrm{DI}}\right) \\
\text { Total de alunos ingressantes }\left(\mathrm{N}_{\mathrm{V}}\right)\end{array}$ \\
\hline
\end{tabular}

Fonte: Decisão TCU n. ${ }^{\circ}$ 408/2002

Nota: $\left(^{*}\right)$ indicadores sem os valores correspondentes ao hospital universitário. As siglas significam $A_{G}$ : Total de alunos regularmente matriculados na graduação; $A_{P G}$ : total de alunos de pós-graduação; $A_{G} E$ : Aluno equivalente de graduação; $A_{G} T I$ : Aluno de graduação em tempo integral; $A_{P G} T I$ : Aluno de pós-graduação em tempo integral; $A_{R} T I$ : Alunos de residência médica em tempo integral; D: Professores doutores; M: Professores mestres; E: Professores especialistas; G: Professores graduados.

Por outro lado, os indicadores de qualidade Índice Geral de Cursos (IGC), Conceito Preliminar de Curso (CPC), Indicador de Diferença entre os Desempenhos Observado e Esperado (IDD), e Exame Nacional de Desempenho de Estudantes (Enade) são mensurados a partir dos indicadores primários detalhados no Quadro 2 a seguir: nota formação geral (FG), nota componente específico (CE), nota geral, indicador de diferença entre os desempenhos observado e esperado (IDD), organização didático pedagógico, infraestrutura, oportunidades ampliação formação, quantidade de professores mestres e doutores, regime de trabalho, indicadores de qualidade dos cursos de graduação, mestrado e doutorado.

O tratamento dos dados foi realizado por meio de métodos estatísticos. As técnicas escolhidas para obtenção das conclusões do estudo foram a análise de correlação e o teste de Spearman . A escolha da correlação de Spearman ocorreu em razão dos dados não terem apresentado distribuição normal.

\section{Resultados e Discussões}

O levantamento documental realizado nos relatórios de gestão evidenciou que as universidades federais brasileiras possuem diferentes níveis de desempenho. A Tabela 1 apresenta a média, o desvio-padrão e 
os valores mínimo e máximo dos indicadores de desempenho das 50 universidades federais pesquisadas. $O$ indicador custo corrente por aluno equivalente, com e sem hospital universitário, apresentam grande dispersão, o valor máximo alcança $R \$ 47.910$, enquanto os valores mínimos alcançam $R \$ 7.078$ e $R \$$ 7.126, respectivamente. Portanto, a estrutura das universidades federais não são uniformes, independente de possuírem hospital universitário ou não.

Tabela 1 - Média, desvio-padrão, valores mínimos e máximos dos indicadores de desempenho das universidades federais. 2015.

\begin{tabular}{lcccc}
\hline Indicadores de desempenho/Indicadores de qualidade & Média & $\begin{array}{c}\text { Desvio- } \\
\text { padrão }\end{array}$ & Mínimo & Máximo \\
\hline Custo corrente com HU/Aluno equivalente (R\$) & 19.931 & 7.078 & 9.532 & 47.910 \\
Custo corrente sem HU/ Aluno equivalente (R\$) & 18.792 & 7.126 & 7.262 & 47.910 \\
Aluno tempo integral/ Professor equivalente & 11,670 & 2,634 & 3,520 & 16,610 \\
Aluno tempo integral / Funcionário equivalente com HU & 6,641 & 2,485 & 2,710 & 14,300 \\
Aluno tempo integral / Funcionário equivalente sem HU & 8,027 & 2,604 & 2,710 & 14,300 \\
Funcionário equivalente com HU / Professor equivalente & 1,891 & 0,577 & 0,880 & 3,710 \\
Funcionário equivalente sem HU / Professor equivalente & 1,514 & 0,330 & 0,880 & 2,750 \\
Grau de participação estudantil (GPE) & 0,742 & 0,132 & 0,370 & 1,090 \\
Grupos de envolvimento discente com pós-graduação (CEPG) & 0,103 & 0,061 & 0,009 & 0,300 \\
Conceito Capes/MEC para pós-graduação & 3,823 & 0,671 & 3,000 & 6,430 \\
Índice de qualificação do corpo docente (IQCD) & 4,244 & 0,379 & 3,440 & 5,000 \\
Taxa de sucesso na graduação (TSG) & 44,610 & 14,484 & 3,98 & 85,84 \\
\hline
\end{tabular}

Fonte: Relatórios de gestão das universidades federais.

Nota: $\mathrm{HU}=$ Hospital Universitário

A média de aluno tempo integral por professor equivalente é de 11,670, com desvio-padrão de 2,634, enquanto o indicador de aluno tempo integral por funcionário equivalente, com e sem hospital universitário, teve média de 6,641 e 8,027, e desvio-padrão de 2,485 e 2,604, respectivamente. Isto é, a quantidade de professor por aluno em tempo integral é superior a quantidade de funcionários por aluno. Já a quantidade de funcionários equivalentes por professor equivalente é de 1,891 e 1.514, em média, respectivamente. Assim, a quantidade média de funcionários é maior que a quantidade média de professores, independente de as universidades possuírem ou não hospital universitário. A maior quantidade média do indicador, quando considerado os hospitais universitários, decorre naturalmente da maior necessidade de profissionais técnicos como enfermeiros, auxiliares de enfermagem, entre outros, para prestação dos serviços aos pacientes.

O grau de participação estudantil (GPE) alcançou média de 0,742 , enquanto o grau de envolvimento dos discentes com a pós-graduação (CEPG) foi de apenas 0,103 . Logo, nota-se que a participação dos alunos é diferente entre os níveis de graduação e pós-graduação. Por outro lado, o conceito Capes/MEC para pós-graduação médio é de 3,823 , enquanto o índice de qualificação do corpo docente tem valor médio alto, de 4,244 , já que o valor máximo é de 5,000. A taxa de sucesso na graduação média é baixa, pois apenas $44,610 \%$ dos alunos conseguem concluir os cursos.

Os indicadores de qualidade das universidades pesquisas foram obtidos na base de dados do INEP, conforme Tabela 2, a qual apresenta média, desvio-padrão, valor mínimo e máximo dos componentes de avaliação da qualidade, a seguir: 
Tabela 2 - Média, desvio-padrão, valor mínimo e máximo dos componentes dos indicadores de qualidade do INEP das universidades federais. 2015.

\begin{tabular}{lccccc}
\hline Indicadores de qualidade & Avaliação & Média & $\begin{array}{c}\text { Desvio- } \\
\text { Padrão }\end{array}$ & Mínimo & Máximo \\
\hline Nota formação geral (FG) & Enade & 3,276 & 0,832 & 0,000 & 5,000 \\
Nota componente específico (CE) & Enade & 3,199 & 0,807 & 0,145 & 5,000 \\
Nota geral & Enade & 3,249 & 0,830 & 0,000 & 5,000 \\
IDD & CPC & 2,324 & 0,655 & 0,000 & 3,401 \\
Organização Didático-Pedagógica & CPC & 1,957 & 0,602 & 0,000 & 3,452 \\
Infraestrutura & CPC & 2,117 & 0,757 & 0,000 & 3,681 \\
Oportunidades ampliação formação & CPC & 2,484 & 0,596 & 0,000 & 3,812 \\
Mestre & CPC & 4,274 & 0,579 & 2,912 & 5,000 \\
Doutor & CPC & 2,892 & 1,045 & 1,180 & 5,000 \\
Regime de trabalho & CPC & 4,976 & 0,115 & 4,355 & 5,000 \\
Graduação & IGC & 2,965 & 0,363 & 2,115 & 3,876 \\
Mestrado & IGC & 4,340 & 0,211 & 4,000 & 4,887 \\
Doutorado & IGC & 3,993 & 1,654 & 0,000 & 4,947 \\
\hline Fon & & & & & \\
\hline
\end{tabular}

Fonte: Inep

Nota: $\mathrm{HU}=$ Hospital Universitário

Os resultados evidenciam que os componentes do Enade, seja a Nota FG, seja a Nota CE, seja a Nota geral, possuem médias próximas de 3,276, 3,199 e 3,249 pontos, respectivamente. Os desviospadrão também são parecidos, em torno de 0,832, 0,807 e 0,830 ponto, respectivamente. Esses resultados em conjunto evidenciam que o nível de aprendizado dos alunos, em formação geral ou em componentes específicos, são semelhantes. Considerando que a nota máxima é de 5,000 pontos, o aproveitamento alcançado pelos alunos das universidades federais, em média, é de $65,52 \%$ para a formação geral e de $63,98 \%$ para os componentes específicos.

A nota média obtida para o Indicador de diferença entre o desempenho observado e esperado (IDD) é de apenas 2,324 pontos. Assim, o valor agregado pelo processo formativo alcançado pelos cursos de graduação oferecidos pelas universidades federais é somente de $46,48 \%$ em termos médios. Os indicadores mensurados a partir da percepção dos estudantes, ou seja, a organização didático-pedagógica, a infraestrutura e instalações físicas, e as oportunidades para ampliação da formação acadêmica e profissional, são baixas. Em termos médios, a organização alcançou 1,957, enquanto a infraestrutura e instalações físicas alcançaram 2,117 pontos e as oportunidades, 2,484 pontos, algo que revela a necessidade de aperfeiçoamento desses requisitos, segundo a percepção dos alunos.

A formação dos professores a nota dos componentes Mestre e Doutor são diferentes em média. Enquanto o componente Mestre alcançou 4,274 pontos, o componente Doutor atingiu apenas 2,892, ou seja, a maior parte dos professores das universidades apresentam, no mínimo, a formação de mestre. Quanto ao regime de trabalho, as universidades federais apresentam indicador alto, com média de 4,976 pontos, haja vista que a grande maioria dos profissionais possuem dedicação exclusiva de trabalho. 
As notas obtidas pelas universidades federais nos componentes do IGC correspondente aos cursos de graduação, mestrado e doutorado, que evidenciam que os melhores cursos oferecidos são os cursos de mestrado, com nota de 4,340 pontos, seguido pelos cursos de doutorado, com 3,993 pontos, e, por último, os cursos de graduação, com 2,965 pontos. Os valores máximo e mínimo do curso de mestrado é 4,000 e 4,887 pontos, respectivamente. Portanto, a qualidade é alta e existe certo nível de uniformidade de qualidade dentre as instituições, diferente do doutorado, que tem maior disparidade. A Tabela 3 apresenta a matriz de correlação de Spearman entre os indicadores de desempenho obtidos nos relatórios de gestão das universidades e os indicadores de qualidade mensurados pelo Inep, conforme segue:

Tabela 3 - Matriz de correlação de Spearman entre indicadores de desempenho e dos componentes dos indicadores de qualidade do Enade das universidades federais. 2015.

\begin{tabular}{lccc}
\hline Indicadores de desempenho/Indicadores de qualidade & Nota FG & Nota CE & Nota Geral \\
\hline Custo corrente com HU/Aluno equivalente & $-0,038$ & 0,032 & $-0,001$ \\
Custo corrente sem HU/ Aluno equivalente & $-0,066$ & 0,003 & $-0,029$ \\
Aluno tempo integral/ Professor equivalente & $0,257^{*}$ & 0,194 & 0,219 \\
Aluno tempo integral / Funcionário equivalente com HU & 0,244 & 0,153 & 0,202 \\
Aluno tempo integral / Funcionário equivalente sem HU & $0,311^{* *}$ & 0,212 & 0,245 \\
Funcionário equivalente com HU / Professor equivalente & $-0,044$ & 0,035 & $-0,015$ \\
Funcionário equivalente sem HU / Professor equivalente & $-0,143$ & $-0,062$ & $-0,104$ \\
Grau de participação estudantil (GPE) & $-0,028$ & $-0,079$ & $-0,054$ \\
Grupos de envolvimento discente com PÓS-GRADUAÇÃO (CEPG) & $0,416^{* * *}$ & $0,459^{* * *}$ & $0,449^{* * *}$ \\
Conceito Capes/MEC para pós-graduação & $0,437^{* * *}$ & $0,409^{* * *}$ & $0,408^{* * *}$ \\
Índice de qualificação do corpo docente (IQCD) & $0,395^{* * *}$ & $0,453^{* * *}$ & $0,440^{* * *}$ \\
Taxa de sucesso na graduação (TSG) & $0,343^{* *}$ & 0,241 & $0,283^{*}$ \\
\hline
\end{tabular}

Fonte: Inep e relatórios de gestão das universidades federais.

Nota: * Coeficiente estatisticamente significativo a $10 \%$; ${ }^{* *} 5 \%$; ${ }^{* *} 1 \%$. HU = Hospital Universitário

A análise dos coeficientes de correlação estatisticamente significativos apresentados na Tabela 3 evidenciam principalmente que o maior envolvimento dos discentes na Pós-Graduação está fortemente correlacionado com melhores notas da formação geral, componentes específicos e nota geral do Enade. Ou seja, parece que os alunos com maior envolvimento na pós-graduação realizam atividades que repercutem na formação da graduação, com atividades de ensino e pesquisa.

Os cursos de pós-graduação com melhores conceitos Capes/MEC estão positivamente correlacionados com as notas do Enade. Esse resultado é interessante, porque evidencia que o fortalecimento da pósgraduação acaba por repercutir também na graduação, já que os professores que participam dos cursos de pós-graduação, em sua grande maioria, são os mesmos que trabalham na graduação. Essa interpretação é consistente, inclusive, porque o IQCD também foi positivamente correlacionado com as notas do Enade, ou seja, a melhor qualificação dos professores resulta em melhores notas.

A taxa de sucesso na graduação também foi positivamente correlacionada com a nota de Formação Geral e Nota Geral do Enade, ou seja, o fomento das condições de permanência do aluno até o final do curso favorece a melhor obtenção de notas dos componentes de avaliação do Enade. 
Em seguida, a Tabela 4 apresenta a matriz de correlação de Spearman entre os indicadores de desempenho obtidos pelas universidades federais e os componentes dos indicadores de qualidade da avaliação do CPC realizado pelo Inep, conforme segue:

Tabela 4 - Matriz de correlação de Spearman entre indicadores de desempenho e dos componentes dos indicadores de qualidade do CPC das universidades federais. 2015.

\begin{tabular}{|c|c|c|c|c|c|c|c|}
\hline $\begin{array}{l}\text { Indicadores de desempenho/ } \\
\text { Indicadores de qualidade }\end{array}$ & IDD & $\begin{array}{l}\text { Didático- } \\
\text {-Pedagó-gico }\end{array}$ & $\begin{array}{l}\text { Infra- } \\
\text { estrutura }\end{array}$ & $\begin{array}{l}\text { Oportu- } \\
\text { nidades }\end{array}$ & Mestre & Doutor & $\begin{array}{c}\text { Regime de } \\
\text { trabalho }\end{array}$ \\
\hline $\begin{array}{l}\text { Custo corrente com HU*/Aluno } \\
\text { equivalente }\end{array}$ & $-0,073$ & 0,080 & 0,022 & 0,130 & $0,257^{*}$ & $0,376^{\star * *}$ & 0,250 \\
\hline $\begin{array}{l}\text { Custo corrente sem HU/ Aluno } \\
\text { equivalente }\end{array}$ & $-0,040$ & 0,133 & 0,071 & 0,161 & $0,309^{* *}$ & $0,352^{* *}$ & $0,304^{* *}$ \\
\hline $\begin{array}{l}\text { Aluno tempo integral/ Professor } \\
\text { equivalente }\end{array}$ & $-0,120$ & $-0,417$ & $-0,105$ & $-0,302$ & $-0,236$ & 0,142 & $-0,346^{* *}$ \\
\hline $\begin{array}{l}\text { Aluno tempo integral / Funcionário } \\
\text { equivalente com HU }\end{array}$ & 0,108 & 0,054 & 0,165 & $-0,219^{*}$ & $-0,019$ & $-0,107$ & $-0,132$ \\
\hline $\begin{array}{l}\text { Aluno tempo integral / Funcionário } \\
\text { equivalente sem HU }\end{array}$ & $-0,233$ & $-0,213$ & $-0,124$ & $-0,353^{* *}$ & $-0,099$ & 0,054 & $-0,295^{\star}$ \\
\hline $\begin{array}{l}\text { Funcionário equivalente com } \mathrm{HU} / \\
\text { Professor equivalente }\end{array}$ & $-0,181$ & $-0,268$ & $-0,177$ & 0,060 & $-0,046$ & $0,281^{* *}$ & $-0,098$ \\
\hline $\begin{array}{l}\text { Funcionário equivalente sem } \mathrm{HU} / \\
\text { Professor equivalente }\end{array}$ & 0,187 & 0,018 & 0,132 & $0,337^{* *}$ & 0,161 & 0,180 & 0,242 \\
\hline $\begin{array}{l}\text { Grau de participação estudantil } \\
\text { (GPE) }\end{array}$ & $-0,069$ & 0,052 & 0,052 & $-0,063$ & $-0,023$ & $-0,119$ & $-0,264^{*}$ \\
\hline $\begin{array}{l}\text { Grupos de envolvimento discente } \\
\text { com pós-graduação (CEPG) }\end{array}$ & $-0,227$ & $-0,195$ & 0,137 & $-0,054$ & 0,148 & $0,413^{* * *}$ & $-0,106$ \\
\hline $\begin{array}{l}\text { Conceito Capes/MEC para pós- } \\
\text { graduação }\end{array}$ & $-0,109$ & $-0,285^{\star}$ & 0,019 & $-0,055$ & 0,099 & $0,344^{* * *}$ & $-0,096$ \\
\hline $\begin{array}{l}\text { Índice de qualificação do corpo } \\
\text { docente (IQCD) }\end{array}$ & 0,230 & $0,355^{\star *}$ & $0,425^{\star * *}$ & $0,411^{* \star *}$ & $0,527^{* * *}$ & $0,742^{* * *}$ & 0,034 \\
\hline $\begin{array}{l}\text { Taxa de sucesso na graduação } \\
\text { (TSG) }\end{array}$ & $-0,101$ & $-0,233$ & $-0,075$ & $-0,195$ & 0,037 & 0,149 & $-0,245$ \\
\hline
\end{tabular}

Fonte: Inep e relatórios de gestão das universidades federais.

Nota: * Coeficiente estatisticamente significativo a $10 \%$; ${ }^{* *} 5 \%$; ${ }^{* *} 1 \%$. HU $=$ Hospital Universitário

Os coeficientes estatisticamente significativos, observados na Tabela 4, apresentam resultados importantes. As correlações positivas entre o custo corrente por aluno equivalente, com e sem hospital universitário, em relação às notas de mestre e doutor é algo esperado, visto que a manutenção de profissionais com maior qualificação requer maior gasto com remuneração na contratação da mão de obra. O custo corrente por aluno equivalente sem hospital universitário foi correlacionado positivamente com o regime de trabalho, pois existe uma maior quantidade de profissionais com dedicação exclusiva nas universidades em comparação com os hospitais universitários.

A correlação positiva entre o índice de qualificação do corpo docente (IQCD) e alguns indicadores (didático-pedagógico, infraestrutura, oportunidades, mestre e doutor) evidencia claramente que a melhor qualificação dos professores resulta em ganhos, tanto em termos de notas dos alunos, conforme foi observado na Tabela 3, como na percepção deles em termos de apoio didático, infraestrutura e de oportunidades de formação. Além disso, é possível notar que a melhoria da formação resulta em melhores indicadores nos quesitos de mestrado e doutorado, ou seja, a formação provavelmente tem sido oferecida nos cursos de pós-graduação. A melhor qualificação dos professores, no caso, de doutorado, tem efeito positivo para um 
maior envolvimento dos alunos na pós-graduação e na melhor conceituação dos cursos pela Capes/MEC já que a quantidade de doutores é um dos requisitos da avaliação.

Outro importante resultado é que a taxa de sucesso na graduação não foi correlacionada estatisticamente com os indicadores de qualidade do CPC. Em outras palavras, isto significa que os motivos que podem ensejar melhor taxa de sucesso na graduação não estão relacionados com a melhoria das condições institucionais, mas, provavelmente, estejam relacionados ao contexto social e individual do aluno.

Na sequência, a Tabela 5 apresenta a matriz de correlação de Spearman entre os indicadores de desempenho e dos componentes da avaliação do IGC das universidades federais.

Tabela 5 - Matriz de correlação de Spearman entre indicadores de desempenho e dos componentes da avaliação de qualidade do IGC das universidades federais. 2015.

\begin{tabular}{lccc}
\hline Indicadores de desempenho/Indicadores de qualidade & Graduação & Mestrado & Doutorado \\
\hline Custo corrente com HU*/Aluno equivalente & $0,284^{* *}$ & 0,078 & 0,032 \\
Custo corrente sem HU/ Aluno equivalente & $0,293^{* *}$ & $-0,046$ & $-0,072$ \\
Aluno tempo integral/ Professor equivalente & 0,053 & $0,494^{* * *}$ & $0,414^{* * *}$ \\
Aluno tempo integral / Funcionário equivalente com HU & $-0,044$ & 0,007 & 0,040 \\
Aluno tempo integral / Funcionário equivalente sem HU & $-0,040$ & $0,337^{* *}$ & $0,273^{*}$ \\
Funcionário equivalente com HU / Professor equivalente & 0,197 & $0,404^{* * *}$ & $0,303^{* *}$ \\
Funcionário equivalente sem HU / Professor equivalente & 0,219 & $-0,072$ & $-0,108$ \\
Grau de participação estudantil (GPE) & $-0,151$ & $-0,153$ & $-0,180$ \\
Grupos de envolvimento discente com pós-graduação (CEPG) & $0,312^{* *}$ & $0,772^{* * *}$ & $0,696^{* * *}$ \\
Conceito Capes/MEC para pós-graduação & 0,345 & $0,811^{* * *}$ & $0,730^{* * *}$ \\
Índice de qualificação do corpo docente (IQCD) & $0,670^{* * *}$ & $0,375^{* * *}$ & $0,375^{* * *}$ \\
Taxa de sucesso na graduação (TSG) & 0,065 & $0,352^{* *}$ & $0,276^{*}$ \\
\hline
\end{tabular}

Fonte: Inep e relatórios de gestão das universidades federais.

Nota: * Coeficiente estatisticamente significativo a $10 \%$; ${ }^{* *} 5 \%$; *** $1 \%$. HU $=$ Hospital Universitário

Na Tabela 5, os coeficientes de correlação estatisticamente significativos apresentam, principalmente, que os custos correntes por aluno equivalente estão correlacionados positivamente com a qualidade dos cursos de graduação, provavelmente por conta da melhor qualificação dos professores, conforme pode ser observado na Tabela 4

O indicador de aluno em tempo integral por professor equivalente foi positivamente correlacionado com melhores indicadores dos cursos de mestrado e doutorado. Esse resultado é esperado, porque, nos cursos de pós-graduação, a maior complexidade dos estudos requer maior dedicação dos professores no suporte aos alunos, de modo que, quanto menor a quantidade de alunos por professor, melhores são os resultados obtidos nos trabalhos de pesquisa.

A correlação positiva entre os indicadores de aluno em tempo integral por funcionário equivalente e funcionário equivalente por professor equivalente com hospital universitário, assim como a não significância estatística do indicador de funcionários equivalentes por professor equivalente sem hospital universitário com as notas dos cursos de mestrado e doutorado, revela que a quantidade de funcionários por aluno em hospitais universitários é importante para obtenção de melhores notas nos cursos de mestrado e doutorado. Provavelmente essa relação seja estabelecida por conta dos cursos de pós-graduação de saúde, em que os funcionários técnicos desempenho papel preponderante em atividades de suporte à pesquisa dos professores ou dos alunos.

O indicador de envolvimento discente com pós-graduação está positivamente correlacionado com maiores notas Nos cursos de graduação, mestrado e doutorado, sendo em maior intensidade no curso de 
mestrado, seguido do doutorado. Esse resultado é esperado, haja vista que os cursos de pós-graduação requerem atividades de pesquisa que necessitam forte dedicação dos alunos. O efeito positivo na graduação supostamente decorre de atividades que envolvem os alunos de graduação em atividades de pesquisa, ensino e extensão desenvolvidos pelos alunos da pós-graduação, como minicursos, palestras, coleta de dados e ações sociais.

Novamente, o índice de qualificação do corpo docente foi correlacionado positivamente aos indicadores de qualidade, no caso específico, dos cursos de graduação, mestrado e doutorado. A maior intensidade da relação é, inclusive, observada com as notas da graduação. Assim, o investimento na qualificação dos professores parece ser, sob todos os aspectos observados da qualidade, um fator preponderante para melhoria da qualidade das universidades federais.

A Figura 1 apresenta o correlograma entre os indicadores de desempenho apurados pelas universidades federais e os indicadores de qualidade medidos pelo Inep. A visão geral das correlações permite observar que a coluna do indicador do IQCD (coluna 11) apresenta cor quase que completamente azul, com exceção, apenas, do regime de trabalho.

Figura 1 - Correlograma entre indicadores de desempenho e de qualidade das universidades federais. 2015.

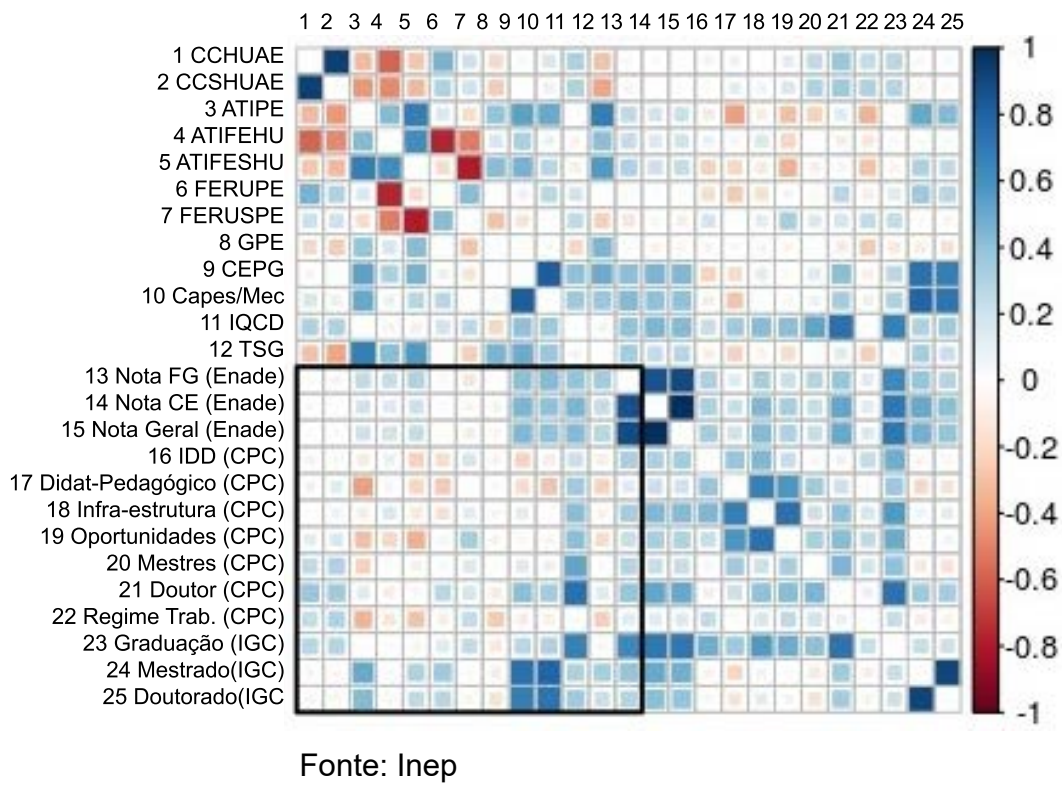

Nesse sentido, a linha do indicador de mestre e doutor (linhas 20 e 21) apresenta cor azul, principalmente, nos indicadores de envolvimento dos discentes da pós-graduação, avaliação da Capes/MEC e No índice de qualidade do corpo docente, conforme esperado a priori. As notas do Enade, nas suas três concepções (de formação geral, componentes específicos e nota geral), são fortemente correlacionadas aos indicadores 9 , 10, 11 e 12, respectivamente, CEPG, Capes/MEC, IQCD, e TSG.

\section{Conclusão}

Os indicadores de desempenho e de qualidade investigados na pesquisa apresentaram correlações positivas estatisticamente. Esses achados refletem que os indicadores de desempenho têm conseguido refletir parcialmente os resultados obtidos pelas universidades federais em relação à qualidade do ensino. Os principais resultados evidenciam a relevância do investimento na qualificação dos professores, a importância do fortalecimento dos programas de pós-graduação para a melhoria da qualidade do ensino, e que o volume de recursos gasto por aluno está diretamente correlacionado com os melhores indicadores do quadro de professores.

Os resultados da pesquisa ainda evidenciam a necessidade de aprofundamento das relações dos indicadores de desempenho e qualidade do ensino superior, principalmente com a identificação dos fatores 
que estimulam o maior envolvimento dos alunos nos programas de pós-graduação, na identificação dos estímulos que influenciam na motivação dos professores em obter melhor qualificação, e nos aspectos que influenciam a percepção dos alunos em relação aos itens: didático-pedagógico, infraestrutura e oferta de oportunidades para formação.

\section{Referências}

AAKER, D. A. Administração estratégica de mercado. 5. ed. Porto Alegre: Bookman, 2001.

BARROS, A. da S. X. Expansão da educação superior no Brasil: limites e possibilidades. Educ. Soc., Campinas, v. 36, n. 131, p. 361-390, abr./jun. 2015.

BEHN, R. D. Why measure performance? Different purpose require different measures. Public Administration Review, Washington, v. 63, n. 5, p. 586-606, Sep./Oct. 2003.

BRASIL. Instituto Nacional de Estudos e Pesquisas Educacionais Anísio Teixeira. Sistema Nacional de Avaliação da Educação Superior (SINAES). Brasília: INEP, 2015.

BRASIL. Portaria no 4.362 de 29 de dez. de 2004. Institui o Banco Único de Avaliadores da Educação Superior do Ministério da Educação, tendo como referência o perfil do docente avaliador. Diário Oficial [da República Federativa do Brasil], Brasília, DF, 30 dez. 2004. Disponível em: http://www.inep.gov.br/ superior/avaliacao_institucional/legislacao.htm. Acesso em: 01 jul. 2017.

BRESSER-PEREIRA, L. C. A reforma gerencial do Estado de 1995. Revista De Administração Pública, Rio de Janeiro, v. 34, n. 4, p. 7-26, 2000.

BRESSER-PEREIRA, L. C. Construindo o Estado republicano: democracia e reforma da gestão pública. Rio de Janeiro: FGV, 2009.

CALLADO, A. L. C.; CALLADO, A. A. C.; ALMEIDA, M. A. A utilização de indicadores de desempenho não-financeiros em organizações agroindustriais: um estudo exploratório. Organizações Rurais \& Agroindustriais, Lavras, v. 10, n. 1, p. 35-48, 2008.

CALLADO, A. L. C.; CALLADO, A. A. C.; ANDRADE, L. P. de. Relações entre indicadores de desempenho: um estudo exploratório em empresas localizadas em Serra Talhada/PE. Revista de Negócios, Blumenau, v. 14, n. 1, p. 100-114, 2009.

CALLADO, A. L. C.; CALLADO, A. A. C.; HOLANDA, F. M. Utilização de indicadores de desempenho no setor hoteleiro de João Pessoa-PB. Turismo-Visão e Ação, Balneário Camboriú, v. 10, n. 1, p. 23-38, 2008.

CATELLI, A.; SANTOS, E. S. Mensurando a criação de valor na gestão pública. Revista de Administração Pública, Rio de Janeiro, v. 38, p. 423-449, maio/jun. 2004.

COLOSSI, N.; CONSENTINO, A.; QUEIROZ, E. G. Mudanças no contexto do ensino superior no Brasil: uma tendência ao ensino colaborativo. Revista da FAE, Curitiba, v. 4, n. 1, 2017.

COSTA, F. L.; CASTANHAR; J. C. Avaliação de programas públicos: desafios conceituais e metodológicos. Revista de Administração Pública, Rio de Janeiro, v. 37, n. 5, p. 969-92, 2003.

CURCINO, G. M.; LEMES, S.; BOTINHA, R. A. Efeito do resultado Abrangente nos Indicadores de desempenho das Companhias Abertas brasileiras. Revista Evidenciação Contábil \& Finanças, João Pessoa, v. 2, n. 3, p. 24-40, 2014.

FOLAN, P.; BROWNE, J. A review of performance measurement: towards performance management. Computers in Industry, [S.I.], v. 56, n. 7, p. 663-680, 2005.

MACEBO, D.; SILVA JR., J. R.; OLIVEIRA, J. F. Reformas e políticas: educação superior e pósgraduação no Brasil. Campinas: Átomo \& Alínea, 2008. v. 1. p. 23-51.

GRATERON, I. R. G. Auditoria de gestão: utilização de indicadores de gestão no setor público. Caderno de Estudos Fipecafi, São Paulo, n. 21, maio/ago. 1999. 
HELOU FILHO, E. A.; OTANI, N. A utilização de indicadores na administração pública: a Lei 12.120/2002 do Estado de Santa Catarina. Revista de Ciências da Administração, Florianópolis, v. 9, n. 17, p. 111131, jan./abr. 2007.

HRONEC, S. M.; ANDERSEN, A. Sinais vitais: usando medidas de desempenho da qualidade, tempo e custos para traçar a rota para o futuro de sua empresa. São Paulo: Makron Books, 1994.

KAPLAN, R. S.; NORTON, D. P. The balanced scorecard: the measures that drive performance. The Harvard Business Review, [S.I.], Jan./Feb. 1992. Disponível em: https://hbr.org/1992/01/the-balancedscorecard-measures-that-drive-performance-2. Acesso em: 21 set. 2019.

KOTANE, I.; KUZMINA-MERLINO, I. Non-financial indicators for evaluation of business activity. European Integration Studies, [S.I.], n. 5, p. 213-219, 2011.

LUNKES, R. J.; GASPARETTO, V.; SCHNORRENBERGER, D. Um Estudo sobre as Funções da Controladoria. Revista de Contabilidade e Organizações, São Paulo, v. 4, n. 10, p. 106-126, 2010.

MACARTHUR, J. B. Performance measures that count: monitoring variables of strategic importance. Journal of Cost Management, [S.I.], v. 10, n. 3, p. 39-45, 1996.

MARENGO, S. T.; DIEHL, C. A. A divulgação de indicadores não financeiros em sites municipais gaúchos. Gestão \& Regionalidade, São Caetano do Sul, v. 27, n. 81, 2011.

MARINI, C. O contexto contemporâneo da administração pública na América Latina. Revista do Serviço Público, Brasília, v. 53, n. 4, p. 31-52, out. 2002.

MARQUEZAN, L. H. F.; DIEHL, C. A.; ALBERTON, J. R. Indicadores não financeiros de avaliação de desempenho: análise de conteúdo em relatórios anuais digitais. Contabilidade, Gestão e Governança, Brasília, v. 16, n. 2, 2013.

MATIAS-PEREIRA, J. A governança corporativa aplicada no setor público brasileiro. Administração Pública e Gestão Social, [S.I.], v. 2, n. 1, p. 109-134, 2010.

NEELY, A. Avaliação do desempenho das empresas: porquê, o quê e como. Lisboa: Caminho, 2002.

NEELY, A. D.; RICHARDS, A. H.; MILLS, J. F.; PLATTS, K. W.; BOURNE, M. C. S. Designing performance measures: a structured approach. International Journal of Operations \& Production Management, [S.I.], v. 17, n. 11, p. 1131-53, 1997.

NEELY, A. Measuring business performance. London: The Economist Books, 1998.

NEELY, A. The performance measurement revolution: why now and what next? International Journal of Operations \& Production Management, [S.I.], v. 19, n. 2, p. 205-28, 1999.

NEELY, A.; GREGORY, M.; PLATTS, K. Performance measurement system design: should process based approaches be adopted? International Journal Production Economics, Amsterdam, v. 46/47, 1996.

POINCELOT, E.; WEGMANN, G. Les motivations des managers utilisant descriteres non financiers: une analyse empirique. Comptabilité Contrôle Audit, [S.I.], v.14, n.1, p. 69-92, 2008.

POLIDORI, M. M. Políticas de avaliação da educação superior brasileira: Provão, SINAES, IDD, CPC, IGC e... outros índices. Avaliação, Campinas, v. 14, n. 2, p. 385-438, jul. 2009.

SILVA, L. C. de; BARTOLUZZIO, A. I. S. de S.; ANJOS, L. C. M. dos. Análise da influência da competição política e de indicadores socioeconômicos no nível de Transparência dos municípios pernambucanos.

Gestão Pública: práticas e desafios, Recife, v. 8, n. 1, p. 1-17, 2017.

SINK, D. S.; TUTTLE, T. C. Planejamento e medição para performance. Rio de Janeiro: Quality Mark, 1993.

VARGAS, S. B. D.; DIEHL, C. A.; AYRES, P. R. R.; MONTEIRO, A. F. Indicadores não-financeiros de avaliação de desempenho: análise de conteúdo em relatórios de administração de empresas de telecomunicações. Sociedade, Contabilidade e Gestão, Rio de Janeiro, v. 11, n. 1, 2016. 
VIGNOCHI, L.; GONÇALO, C. R.; LEZANA, Á. G. R. Como gestores hospitalares utilizam indicadores de desempenho? Rev. adm. empres., São Paulo, v. 54, n. 5, p. 496-509, Oct. 2014.

Submetido em: 07/09/2018

Aprovado em: 06/05/2019 